\title{
Observation on therapeutic efficacy of rt-PA intravenous thrombolysis combined with compound anisodine injection on central retinal artery occlusion
}

\author{
XIAO-JUN WU, FENG GAO, XU LIU and QING ZHAO \\ Department of Ophthalmology, Xiangyang Hospital Affiliated to Hubei University of Medicine, \\ Xiangyang, Hubei 441000, P.R. China
}

Received February 5, 2016; Accepted August 22, 2016

DOI: $10.3892 /$ etm.2016.3681

\begin{abstract}
The aim of the present study was to observe the clinical efficacy and safety of recombinant tissue plasminogen activator (rt-PA) combined with compound anisodine in treating central retinal artery occlusion (CRAO). Forty-eight patients diagnosed with CRAO were randomly divided into a treatment group (24 cases) and a control group (24 cases). For the control group, nitroglycerin, 654-2, methazolamide, puerarin and compound anisodine were used for the treatment, along with oxygen, massage and other conventional treatments. Besides conventional therapy, the treatment group was also given intravenous rt-PA thrombolysis. Visual acuity, fundus oculi, visual field changes were taken as indicators for efficacy evaluation. It was found that the total effective rate of the control group was $70.83 \%$, while that for the treatment group was $91.67 \%$, and the comparative difference between the two groups was of statistical significance $(p<0.05)$. The visual field defect of the control group after treatment was approximately $74.26 \pm 12.91 \%$, and the visual field defect of the treatment group after treatment approximately $35.08 \pm 16.33 \%$; thus, the comparative difference was statistically significant $(\mathrm{p}<0.01)$. The comparative difference of the original contents of fibrous protein in blood in the treatment group before and after treatment was statistically significant $(\mathrm{p}<0.01)$. In conclusion, the result show that intravenous thrombolysis with rt-PA combined with compound anisodine is safe and effective in treating CRAO, which can significantly improve the prognosis of patients.
\end{abstract}

Correspondence to: Dr Feng Gao, Department of Ophthalmology, Xiangyang Hospital Affiliated to Hubei University of Medicine, 15 Jiefang Road, Xiangyang, Hubei 441000, P.R. China

E-mail: gao_feng_1212@163.com

Key words: recombinant tissue plasminogen activator, compound anisodine, intravenous thrombolysis, acute central retinal artery occlusion

\section{Introduction}

Central retinal artery occlusion (CRAO) refers to acute retinal ischemia and hypoxia as well as serious damage of visual function caused by disorders in retinal blood circulation, and the disorders result from retinal artery spasm, artery intimitis, or the occlusion in the fiber thrombus of blood platelets or blood vessels (1). Retinal central artery occlusion is one of the emergent cases in ophthalmology that can seriously damage vision and result in unsatisfactory prognosis. Once the duration of retinal ischemia exceeds $90 \mathrm{~min}$, the death of the photoreceptor becomes irreversible.

At present, conventional methods including oxygen inhalation, eyeball massage, tube expansion and anticoagulation are mostly used in clinics, and auxiliary medicine such as vitamin B and energy are administered simultaneously. The curative effects generally fail to be satisfactory (2). Urokinase artery intervention thrombolysis is an emergent technology in the recent decade and is widely used in China. However, the intervention thrombolysis equipment is complex and costly, posing high demands for operations; preoperative preparation time is prolonged by 40-60 min compared to that of intravenous thrombolysis; and hospitals with superselective thrombolysis and mechanical thrombus maceration are extremely limited in China. In addition, urokinase, a non-selective fibrinolytic agent, can enable plasminogen into thrombus and plasma to be activated leading to high-fiber hemolysis of the whole body, which causes complications such as extensive mucocutaneous hemorrhage all over the body (3). By contrast, intravenous thrombolysis is advantageous for its simplicity, safety and is easy to use. Given that the central nervous system is extremely sensitive to ischemia and hypoxia and the retina belongs to the outer reach of the cerebral central nervous system, recombinant tissue plasminogen activator (rt-PA), a thrombolytic drug for treating acute cerebral infarction was selected. rt-PA is a kind of thrombolytic drug featured by high selection, short half-life and no antigenicity (4). It has high affinity to fibrous protein in plasma, enabling the lysine in the compounds to be activated. At the same time, it can induce plasminogen to be turned into plasmin, so that insoluble fibrous protein can be decomposed into soluble fibrous protein fragments and local thrombolysis can be promotetd. However, it has little affinity 
to the plasminogen of the whole coagulation system and exerts inconspicuous fibrinolysis for the whole body, thus, no hemorrhage tendency occurs.

In the present study, 12 cases of CRAO patients having attacks within $24 \mathrm{~h}$ were treated with intravenous medicine administration with $\mathrm{rt}-\mathrm{PA}(0.9 \mathrm{mg} / \mathrm{kg}$, the highest dose being $90 \mathrm{mg}$ ).

\section{Materials and methods}

General information. Forty-eight cases (48 eyes) of acute CRAO patients (5) diagnosed at the Xiangyang Hospital Affiliated to Hubei University of Medicine (Hubei, China) from June, 2009 to June, 2012 were selected and randomly divided into a treatment group including 24 cases ( 24 eyes) and a control group including 24 cases (24 eyes). None of the cases had received emergency treatment prior to being hospitalized. Exclusion standards for the treatment group were: i) a tendency of active hemorrhage and a known tendency of bleeding; ii) a history of cerebral infarction or myocardial infarction in recent 6 months; iii) current adoption of anticoagulation; iv) intracranial arterial aneurysm or arteriovenous malformation; v) serious heart failure and dysfunction of liver and kidney; vi) the count of thrombocyte being < $100 \times 10^{9} / 1(6,7)$; and vii) gestation and non-cooperation. Among patients in the treatment group, 24 cases were diagnosed with central retinal artery occlusion, including 16 cases of males and 8 cases of females as well as 14 cases of right eyes and 10 cases of left eyes. Their ages ranged from 42 to 76 years, with an average of 58.8 years. In terms of the duration between the attack and visiting a doctor, there were 4 cases $<2$ h, 12 cases between 2-6 h, 5 cases between 6-24 h, 2 cases around 24-72 h, and 1 case with $>72 \mathrm{~h}$. Fourteen cases had hypertension and arteriosclerotic disease, 8 cases had transient cerebral ischemia and 8 cases had cerebral thrombosis. Among patients in the control group, 24 cases were diagnosed with central retinal artery occlusion including 14 cases of males and 10 cases of females as well as 17 cases of right eyes and 7 cases of left eyes. The ages of those patients ranged from 36 to 72 , with an average of 50.2 years. In terms of the duration between the attack and visiting a doctor, there were 3 cases for $<2 \mathrm{~h}, 9$ cases between 2-6 h, 9 cases between 6-24 h, 1 case between 24-72 h, 2 cases were $>72 \mathrm{~h}$. Sixteen cases had a history of hypertension and arteriosclerotic disease, 5 cases had a history of diabetes, and 3 cases had a history of cerebral thrombosis. The comparative difference of general information between the two groups was of no statistical significance.

Treatment methods. Treatment groups were divided as follows: group 1 were given $0.5 \mathrm{mg}$ of sublingual nitroglycerin tablets immediately melted 3 times a day. Once per day, retrobulbarly was injected with $0.5 \mathrm{ml}$ of atropine, and was orally administered with $50 \mathrm{mg}$ of methazolamide tablets twice a day. The cases inhaled oxygen (the gas mixture of oxygen with a volume fraction of $95 \%$ and carbon dioxide with a volume fraction of $5 \%$ ) for $10 \mathrm{~min}$ every $1 \mathrm{~h}$, eyeballs were massaged repetitively and intermittently, alternatively eyeballs were pressed on the patients' upper palpebras with index fingers of both hands for $10-15$ sec each time $(8,9)$.
Group 2 were administered with rt-PA within 30 mins $\left(0.5 \mathrm{mg} \cdot \mathrm{kg}^{-1}\right.$; the maximum dosage being $90 \mathrm{mg}$ ), initially $10 \%$ of the dose was injected through veins and the $90 \%$ via intravenous drip within $1 \mathrm{~h}$. The signs were monitored and observed for mucocutaneous, respiratory and archenteric hemorrhage or bleeding in the urinary tract every $0.5 \mathrm{~h}$ within $3 \mathrm{~h}$ after thrombolysis, and then monitored once every hour within $24 \mathrm{~h}$. Anticoagulation and antiplatelet agents were forbidden within $24 \mathrm{~h}$. If no hemorrhage occurred, $80 \mathrm{mg}$ of sodium ozagrel was given and $100 \mathrm{ml}$ of $9 \mathrm{~g} \cdot \mathrm{l}^{-1}(10-13)$. Sodium chloride injection was added, which was adopted twice a day through intravenous drip. If any tendency of hemorrhage was identified, the medicine was immediately stopped and antifibrinolytic agents given, and fresh plasma or blood was transfused if necessary.

Group 3: these cases were simultaneously injected with $2 \mathrm{ml}$ of compound anisodine subcutaneously beside the lateral superficial temporal artery twice a day, and continued for 2 courses of treatment with 14 days being one course.

Group 4: these cases were orally administered with $0.5 \mathrm{~g}$ of mecobalamin tablets three times a day and injected intramuscularly $0.1 \mathrm{~g}$ vitamin B1 and $0.5 \mathrm{mg}$ of B12. Days (13 or 14) after medications, visual acuity, fundus oculi, and visual field were monitored; hemostasis and hepatorenal function were respectively checked $24 \mathrm{~h}$ and 7 days after the treatment. The treatment in the treatment group lasted 14-52 days, with an average period of 38 days (14-16).

Control groups were divided as follows: group 1, immediate eyeball massage as well as oxygen inhalation was given, and patients were asked to have melted sublingual nitroglycerin. Retrobulbarly, $0.5 \mathrm{ml}$ of atropine was injected once a day, and $50 \mathrm{mg}$ of methazolamide tablets were orally administered twice a day. The course of treatment lasted for 1 week.

Group 2: these cases were given $0.4 \mathrm{~g}$ of puerarin injection within half an hour and intravenous drip of $250 \mathrm{ml}$ of $50 \mathrm{~g} \cdot \mathrm{l}^{-1}$ glucose injection or $250 \mathrm{ml}$ of $9 \mathrm{~g} \cdot \mathrm{l}^{-1}$ sodium chloride injection was provided once a day, and one course of treatment lasted for 2 weeks (17-19).

Group 3: these cases were simultaneously injected with $2 \mathrm{ml}$ of compound anisodine subcutaneously beside the lateral superficial temporal artery twice a day for 2 courses of treatment with one course being 14 days.

Group 4: these cases were orally administered with $0.1 \mathrm{~g}$ of aspirin enteric-coated tablets once a day, orally administered $0.5 \mathrm{~g}$ of mecobalamine tablets three times a day, intramuscularly injected with $0.1 \mathrm{~g}$ of vitamins B1 and B12. The treatment in the control group lasted for 15-58 days, with an average period of 39 days (20-22).

Observation indices. Follow-up was conducted for an average of 3 months to examine visual acuity, fundus oculi and visual field to monitor fibrinogen changes and observe curative effects. International standard visual acuity chart was adopted in vision check and Oculus fully automatic perimeter was adopted in checking visual field.

Evaluation of curative effects. Curative effects could be classified into four levels on the basis of the situations of visual acuity, fundus oculi and visual field as: cured, markedly effective, effective and ineffective, respectively. Cured indicated that 
Table I. Comparison of the clinical efficacy of the two groups (n, \%).

\begin{tabular}{lcccccc}
\hline Group & $\mathrm{n}$ & Cured & Markedly effective & Effective & Ineffective & Total effective \\
\hline Treatment & 24 & $3(12.5)$ & $13(54.16)$ & $6(25)$ & $2(8.3)$ & $22(91.67)$ \\
Control & 24 & $1(4.17)$ & $6(25)$ & $10(41.67)$ & $7(29.17)$ & $17(70.83)$ \\
\hline
\end{tabular}

Table II. Comparison of visual acuity between two groups before and after treatment (eye).

\begin{tabular}{lccccccc}
\hline & & \multicolumn{5}{c}{ Before/after treatment } \\
\cline { 3 - 8 } Group & $\mathrm{n}$ & No LP & LP-0.02 & $0.02-0.1$ & $0.1-0.3$ & $0.3-0.5$ & $>0.5$ \\
\hline Treatment & 24 & $1 / 1$ & $13 / 2$ & $5 / 4$ & $3 / 6$ & $2 / 7$ & $0 / 4$ \\
Control & 24 & $0 / 0$ & $11 / 8$ & $8 / 5$ & $4 / 6$ & $1 / 3$ & $0 / 2$ \\
\hline
\end{tabular}

the visual acuity improved by 4 lines or more in the logarithmic visual acuity chart or the visual acuity improved by less than 4.8; no edema existed in the retina of fundus oculi; and the scope of visual field defect reduced by $>50 \%$ or the visual field returns to normal on the whole $(2,21,23-25)$. Markedly effective indicated that the visual acuity improved by $>3$ lines; ischemia of fundus oculi improved basically, and the scope of visual field defect decreased by 30-40\%. Effective indicated that the visual acuity improves by $>2$ lines; mild edema existed in the retina of fundus oculi; ischemia was limited to some degree; and the scope of visual field defect reduced by $20-30 \%$ (20). Ineffective, shows no improvement of visual acuity occurred; wide ischemia was evident in the fundus oculi; and the reduction of the scope of visual field defect was $<10 \%$ (24). Visual acuity $<0.1$ was judged as $0.08,0.06,0.04$ and 0.02 with finger counting, hand movement, light perception and no light perception before eyes taken as 1 , respectively.

Statistical analysis. SPSS 17.0 statistical software (Chicago, IL, USA) was used to process the data. Experimental results are presented as mean \pm standard deviation; enumeration data were examined with $\chi^{2}$ and measurement data were examined with Student's t-test. $\mathrm{P}<0.05$ was considered as statistically significant difference.

\section{Results}

General curative effects. Tables I and II show the comparison of vision and curative effects between the two groups before and after treatment. The results showed that, after treatment, the vision of the treatment group was obviously better than that of the control group $(\mathrm{p}<0.05)$, and the curative efficacy in the treatment group was obviously better than that in the control group $(\mathrm{p}<0.05)$.

Changes of fundus oculi. Before treatment, the patient central retinal artery was obviously turned thin and narrow, the veins were in segments, abundant incanus edema existed behind their retinas, and macula lutea as well as cherry-red spots existed. One week after treatment, blood flow in the central retinal artery was obviously restored for the treatment group, yet edema still existed in the inner layer of the retina and cherry-red spots were evident. While no obvious changes were detected in the examination of fundus oculi before or after treatment for the control group. Three to four weeks after treatment, the edema on the inner layer of posterior pole retina was gradually absorbed, the nerve fiber layer of optic disk and retina partially atrophied, vascular white sheath were visible in part of the central retinal artery and veins. As for the examination of the control group, edema on the inner layer of retina was alleviated, the central retinal artery was still obviously thin, stasis existed in the veins and linea alba were visible in the veins.

Changes of visual field. The visual field defects of the control group was approximately $74.26 \pm 12.91 \%$. The visual field of the treatment group was obviously expanded, and the visual field defects reduced to $35.08 \pm 16.33 \%$. The difference between the treatment group and control group in changes of visual field defects was of statistical significance $(\mathrm{p}<0.05)$.

Changes of fibrinogen. The content of fibrinogen for the treatment group was $3.62 \pm 0.65 \mathrm{~g} \cdot \mathrm{l}^{-1}$ before treatment and $2.81 \pm 0.48 \mathrm{~g} \cdot l^{-1} 24 \mathrm{~h}$ after using rt-PA, the difference in content of fibrinogen for treatment group before and after was statistically significant $(\mathrm{p}<0.01)$. The content of fibrinogen for the control group was $3.94 \pm 0.72 \mathrm{~g} \cdot \mathrm{l}^{-1}$ before treatment and $3.73 \pm 0.59 \mathrm{~g} \cdot \mathrm{l}^{-1}$ after using rt-PA, the difference in the content of fibrinogen of the control group before and after treatment was of not statistically significant $(p>0.05)$. Comparative difference in fibrinogen content between the two groups before treatment was not statistically significant $(p>0.05)$, while the fibrinogen contents of the two groups after treatment was significant $(\mathrm{p}<0.001)$.

Adverse reactions. Three cases (12.5\%) among the patients in treatment group in this group of cases had gum bleeding after being given intravenous thrombolysis, the thrombolysis was not stopped, although the dripping speed was slowed, and there was no serious complication or death. 


\section{Discussion}

rt-PA is approved by American Food and Drug Administration and is the only one attested by a large number of clinical experiments in European and American countries (23). As a thrombolytic drug recommended for use to treat ischemic stroke, it can be administered through arteries or veins. A number of clinical experiments proved that intravenous medication would not add bleeding complications, and Wardlaw et al (7) found that rt-PA was safe and effective after summarizing conditions of intra-arterial and intravenous thrombolysis in China in the recent decade. In recent years, through clinical research covering treatment of acute cerebral infarction, myocardial infarction and acute retinal central artery occlusion in China and other countries (24), it was verified that rt-PA intravenous thrombolysis could significantly improve blood supply to acute ischemic cerebral and cardiac tissues and narrow the range of infarction so that it could reduce the disability rate and death rate caused by acute cardiac and cerebral infarction. Findings showed that rt-PA intravenous thrombolysis could improve patients' visual acuity by at least 3 lines within $24 \mathrm{~h}$ since the attacks of symptoms, and effectively improve as well as store their intraocular perfusion and visual function (27). In the present study, 12 cases of CRAO patients having attacks within $24 \mathrm{~h}$ were treated by intravenous medicine administration with rt-PA $(0.9 \mathrm{mg} / \mathrm{kg}$, the highest dosage being $90 \mathrm{mg}$ ). Ten percent of dosage ictus was initially administered, the remaining dosage was given by continuous intravenous drip for $1 \mathrm{~h}$ continuously, and warfarin anticoagulant therapy was conducted for 1 month. As a result, 10 cases $(83.3 \%)$ witnessed improvement in visual acuity, with 4 cases for 8 lines and 6 cases for 2-4 lines as well as 2 cases with no efficacy, no systematic cerebral complications occurred during the treatment. Nowak et al (13) reported that CRAO patients could significantly improve their central vision and alleviate macular oedema as well as timely remove embolus through intravenous application of rt-PA $4.5 \mathrm{~h}$ following the occurence of symptoms; no embolus was found in sclerous blood vessels during color ultrasonography of bilateral carotid arteries and retinal macular oedema basically disappeared during fundus oculi examination 10 days later. Hazin et al (12) maintained that rt-PA intravenous thrombolytic therapy would be taken as a feasible therapy for treating CRAO, which would benefit patients' visual acuity and fundus oculi conditions when combined with other conventional therapies. Mach and Procházka (15) treated 10 cases of patients with thrombolytic therapy. As a result, 2/3 patients significantly improved their visual acuity after thrombolysis within $12 \mathrm{~h}$; some patients undergoing throbolysis after $>30 \mathrm{~h}$ had their visual acuity improved temporarily despite its late decrease. Therefore, thrombolytic effects for patients with CRAO for $>30 \mathrm{~h}$ would not be very good, and earlier throbolytic therapy is needed.

Compound anisodine is a kind of alkaloid separated from anisodus tanguticus, a plant of solanaceae. As a new preparation for treating ocular ischemic diseases, it is now widely used in ophthalmology clinics. Mainly composed of anisodine and procaine, it is injected intramuscularly to vegetative nerve endings under the skin beside the superficial temporal arteries. It can regulate vasomotor function and nutrition metabolism in the ocular ischemia area, ease vasospasm in the eye and increase the amount of blood flow inside the eyes, in order to improve the blood supply and oxygen supply of the optic nerves in fundus oculi and improve visual function by promoting absorption of exudation from edema. Simultaneously, it can adjust the activity of ocular vegetative nerves, resist oxidation, repress the increase of free calcium and remove oxygen radicals in the cells, prevent and alleviate the occurrence of ischemia-reperfusion injuries, control inflammation and fibroplasia; it can also protect endothelial cytomembrane, restore normal permeability of vascular walls, and promote the organization as well as absorption of edema, hemorrhage and exudation $(28,29)$.

For the 48 cases of CRAO patients in the treatment and control groups, their visual acuity, fundus oculi, and visual field were improved to some extent, while the treatment group exhibited more significant changes. A comparison between the total effective rate of the clinical efficacy treatment group $(91.67 \%)$ and that of the control group (70.83\%) proved that the distinction was of statistical significance $(\mathrm{p}<0.05)$. The visual field defect of the control group was approximately $74.26 \pm 12.91 \%$ and that of the treatment group decreased to $35.08 \pm 16.33 \%$, so the distinction between the two groups was of obvious statistical significance $(\mathrm{p}<0.01)$. Observation results of the cases manifested that: i) therapeutic efficacy was related to the time since the onset of the disease, the extent of visual impairment, the location of angiemphraxis, the size of occluded blood vessels and the part occluded; ii) the shorter the time since the onset of the disease, the better the remaining sight and the efficacy; iii) the shorter the time window of thrombolysis, the better the curative effects, and the efficacy was most remarkable within $6.5 \mathrm{~h}$; and iv) efficacy on branch retinal artery occlusion was better than that on central retinal artery occlusion, which was in agreement with relevant research data (16).

The observation results proved that administration of rt-PA early-phase intravenous thrombolysis together with compound anisodine in treating CRAO could rapidly restore perfusion of ischemic retina as well as alleviate reperfusion injury caused by free radicals, so that CRAO patients' visual nerve function could be remarkably improved. The strategy of combining thrombolysis and protection of visual nerves is of significant efficacy, displaying extremely powerful protection of visual nerves. In addition, for CRAO patients with hypertension, treatment should not reduce blood pressure too quickly when reducing eye pressure, and relatively high perfusion pressure should be retained in the earlier stage to improve the amount of retinal blood flow.

As studies on evidence for rt-PA thrombolysis in treating CRAO remained insufficient, a combination treatment group (rt-pA + compound anisodine) was added in rt-PA intravenous thrombolysis to further prove the conclusion that combination therapy was better than pure conservative therapy. For high doses and long-time use of rt-PA easily add risks of hemorrhage (17), thus rt-PA thrombolysis treatment was applied for carefully selected patients in the cases of this group, and the dosage of rt-PA thrombolysis was set as $0.5 \mathrm{mg} / \mathrm{kg}$ according to previous studies $(10,11)$. It was found that the difference between the contents of fibrinogen in blood for the treatment group before and after treatment was of significance $(\mathrm{p}<0.01)$, 
no drug withdrawal occurred due to hepatorenal function damages, allergy, shock and other adverse reactions to medicine; no serious complication or death occurred after treatment except 3 cases of gingival bleeding (12.5\%). Application of rt-PA to treat CRAO was proved secure and reliable, which could significantly improve the recovery rate, the remarkably effective rate and the effective rate. Therefore, early, timely, precise and effective therapeutic methods are crucial for restoring retinal blood circulation and improving the visual function of fundus oculi.

\section{References}

1. Yen JC, Lin HL, Hsu CA, Li YC and Hsu MH: Atrial fibrillation and coronary artery disease as risk factors of retinal artery occlusion: a nationwide population-based study. BioMed Res Int 2015: 374616, 2015.

2. Antoun JS, Slim EA, Hemade AI, Awada S, Fakhoury HW and Cherfan CG: Idiopathic central retinal artery occlusion in an eight-year-old girl. J Fr Ophtalmol 39: e1-e3, 2016.

3. Naff NJ, Carhuapoma JR, Williams MA, Bhardwaj A, Ulatowski JA, Bederson J, Bullock R, Schmutzhard E, Pfausler B, Keyl PM, Tuhrim S and Hanley DF: Treatment of intraventricular hemorrhage with urokinase : Effects on 30-Day survival. Stroke 31: 841-847, 2000

4. Wardlaw JM, Murray V, Berge E, Del ZG, Sandercock P, Lindley RL and Cohen G: Recombinant tissue plasminogen activator for acute ischaemic stroke: An updated systematic review and meta-analysis. Lancet 379: 2364-2372, 2012.

5. Schmidt DP, Schulte-Mönting J and Schumacher M: Prognosis of central retinal artery occlusion: local intraarterial fibrinolysis versus conservative treatment. AJNR Am J Neuroradiol 23 . 1301-1307, 2002.

6. Hayreh SS and Zimmerman MB: Central retinal artery occlusion: visual outcome. Am J Ophthalmol 140: 376-391, 2005.

7. Wardlaw JM, Murray V, Berge E and Del Zoppo GJ: Thrombolysis for acute ischaemic stroke. Cochrane Database Syst Rev 7 : CD000213, 2009.

8. Yokoyama S, Manabe Y, Fujii D, Ikeda-Sakai Y, Narai H, Omori $\mathrm{N}$ and Abe $\mathrm{K}$ : Intravenous tissue plasminogen activator therapy for an acute ischemic stroke patient with later diagnosed unilateral moyamoya syndrome. J Stroke Cerebrovasc Dis 22: $1190-1192,2013$

9. Zhou ZG, Wang RL and Yu KL: Myocardial infarction following recombinant tissue plasminogen activator treatment for acute ischemic stroke: a dangerous complication. Chin Med J (Engl) 125: 2775-2776, 2012.

10. Hattenbach LO, Kuhli-Hattenbach C, Scharrer I and Baatz H: Intravenous thrombolysis with low-dose recombinant tissue plasminogen activator in central retinal artery occlusion. Am J Ophthalmol 146: 700-706, 2008

11. Chen CS, Lee AW, Campbell B, Lee T, Paine M, Fraser C, Grigg J and Markus R: Efficacy of intravenous tissue-type plasminogen activator in central retinal artery occlusion: report from a randomized, controlled trial. Stroke 42: 2229-2234, 2011.

12. Kattah JC, Wang DZ and Reddy C: Intravenous recombinant tissue-type plasminogen activator thrombolysis in treatment of central retinal artery occlusion. Arch Ophthalmol 120 1234-1236, 2002.
13. Nowak RJ, Amin H, Robeson K and Schindler JL: Acute central retinal artery occlusion treated with intravenous recombinant tissue plasminogen activator. J Stroke Cerebrovasc Dis 21: 913.e5-913.e8, 2012

14. Hazin R, Dixon JA and Bhatti MT: Thrombolytic therapy in central retinal artery occlusion: cutting edge therapy, standard of care therapy, or impractical therapy? Curr Opin Ophthalmol 20: 210-218, 2009.

15. Mach R and Procházka J: Thrombolysis in central retinal artery occlusion using alteplase. Cesk Slov Oftalmol 64: 245-248, 2008 (In Czech)

16. Ma J, Yang X and Li Z: Research progess on introducing thrombolytic therapy into treating retinal central artery occlusion. Int Rev Opthalmology 25: 1-4, 2001.

17. Sugiura S, Iwaisako K, Toyota S and Takimoto H: Simultaneous treatment with intravenous recombinant tissue plasminogen activator and endovascular therapy for acute ischemic stroke within 3 hours of onset. AJNR Am J Neuroradiol 29: 1061-1066, 2008.

18. Wi JM and Chi M: Rhabdomyolysis presenting as orbital apex syndrome. J Craniofac Surg 27: e48-e50, 2016

19. Rodríguez Villa S, Salazar Méndez R, Cubillas Martín M and Cuesta García M: Central retinal artery occlusion after phacoemulsification under peribulbar anaesthesia: pathogenic hypothesis. Arch Soc Esp Oftalmol 91: 40-43, 2016 (In Spanish).

20. Chavala SH, Williamson JF and Postel EA: Embolic central retinal artery occlusion after subcutaneous auricular steroid injection. Lancet: Nov 26, 2015 (Epub ahead of print).

21. Ahn SJ, Park KH, Ryoo NK, Hong JH, Jung C, Yoon $\mathrm{CH}$, Han MK and Woo SJ: No-reflow phenomenon in central retinal artery occlusion: incidence, risk factors, and clinical implications. PLoS One 10: e 0142852, 2015.

22. Rajabi MT, Naderan M, Mohammadi SZ and Rajabi MB: Central retinal artery occlusion following orbital tumor resection: is rapid intervention effective? Indian J Ophthalmol 63: 678-680, 2015.

23. Lu CW, Wang J, Zhou DD, Hao JL, Liang LL, Li XH and Hui P: Central retinal artery occlusion associated with persistent truncus arteriosus and single atrium: a case report. BMC Ophthalmol 15: 137,2015 .

24. Miller NR: Should patients with acute central retinal artery occlusion be treated with intra-arterial t-PA?: Comment. J Neuroophthalmol 35: 444, 2015.

25. Beck KD and Hernandez L: Central retinal artery occlusion in a 21-year-old boxer. Ophthalmology 122: 1568, 2015.

26. de Castro-Abeger AH, de Carlo TE, Duker JS and Baumal CR: Optical coherence tomography angiography compared to fluorescein angiography in branch retinal artery occlusion. Ophthalmic Surg Lasers Imaging Retina 46: 1052-1054, 2015.

27. Sharma M, Prashar A, Tuli R, Sharma RK and Mahajan VK: Atypical central retinal artery occlusion: an uncommon cause of retinopathy and visual loss in dermatomyositis. Int J Rheum Dis: Sep 25, 2015 (Epub ahead of print).

28. Liu WD, Chen LL, Shen CY and Jiang LB: Neuroprotective effect of compound anisodine in a mouse model with chronic ocular hypertension. Chin Med J (Engl) 128: 2652-2657, 2015.

29. Zhu Y, Song C and Wang S: [The changes of hemodynamics in ocular trauma and treatment with compound anisodine]. Zhonghua Yan Ke Za Zhi 32: 110-113, 1996. 